Research paper

\title{
SARS-CoV2 vertical transmission with adverse effects on the newborn revealed through integrated immunohistochemical, electron microscopy and molecular analyses of Placenta
}

\author{
Fabio Facchettia,*,\#, Mattia Bugatti ${ }^{\mathrm{a}, \#}$, Emma Drera $^{\mathrm{a}}$, Claudio Tripodo ${ }^{\mathrm{b}}$, Enrico Sartoric ${ }^{\mathrm{c}}$, \\ Valeria Cancila ${ }^{\mathrm{b}}$, Marta Papaccioc ${ }^{\mathrm{c}}$, Roberta Castellani ${ }^{\mathrm{c}}$, Stefano Casola ${ }^{\mathrm{d}}$, \\ Maria Beatrice Boniotti ${ }^{\mathrm{e}}$, Patrizia Cavadini ${ }^{\mathrm{e}}$, Antonio Lavazza ${ }^{\mathrm{e}, \#}$ \\ ${ }^{a}$ Pathology Unit, Department of Molecular and Translational Medicine, University of Brescia, 25123, Brescia, Italy \\ b Tumor Immunology Unit, Department of Health Sciences, University of Palermo School of Medicine, 90134, Palermo, Italy \\ ${ }^{\mathrm{c}}$ Department of Obstetrics and Gynaecology, University of Brescia, 25123, Brescia, Italy \\ d The FIRC Institute of Molecular Oncology (IFOM), 20139, Milan, Italy \\ e Istituto Zooprofilattico Sperimentale della Lombardia e dell'Emilia Romagna (I.Z.S.L.E.R.), 25124 Brescia, Italy
}

\section{A R T I C L E I N F O}

\section{Article History:}

Received 18 June 2020

Revised 27 July 2020

Accepted 28 July 2020

Available online $\mathrm{xxx}$

\begin{abstract}
A B S T R A C T
Background: . The occurrence of trans-placental transmission of severe acute respiratory syndrome corona virus 2 (SARS-CoV-2) infection remains highly debated. Placental positivity for SARS-CoV-2 has been reported in selected cases, but infection or virus-associated disease of fetal tissues or newborns remains to be demonstrated.

Methods: We screened for SARS-CoV-2 spike (S) protein expression placentas from 101 women who delivered between February 7 and May 15, 2020, including 15 tested positive for SARS-CoV-2 RNA, 34 tested negative, and 52 not evaluated as they did not meet testing criteria (32), or delivered before COVID-19 pandemic declaration (20). Immunostain for SARS-CoV-2 nucleocapsid (N) was performed in the placentas of all COVID-19 positive women. One placenta resulted positive for the SARS-CoV-2 S and N proteins, which was further studied by RNA-in situ hybridization and RT-PCR for S transcripts, and by electron microscopy. A comprehensive immunohistochemical and immunofluorescence analysis of the placental inflammatory infiltrate completed the investigations.

Findings: SARS-CoV-2 S and N proteins were strongly expressed in the placenta of a COVID-19 pregnant woman whose newborn tested positive for viral RNA and developed COVID-19 pneumonia soon after birth. SARS-CoV-2 antigens, RNA and/or particles morphologically consistent with coronavirus were identified in villous syncytiotrophoblast, endothelial cells, fibroblasts, in maternal macrophages, and in Hofbauer cells and fetal intravascular mononuclear cells. The placenta intervillous inflammatory infiltrate consisted of neutrophils and monocyte-macrophages expressing activation markers. Absence of villitis was associated with an increase in the number of Hofbauer cells, which expressed PD-L1. Scattered neutrophil extracellular traps (NETs) were identified by immunofluorescence.

Interpretation: We provide first-time evidence for maternal-fetal transmission of SARS-CoV-2, likely propagated by circulating virus-infected fetal mononuclear cells. Placenta infection was associated with recruitment of maternal inflammatory cells in the intervillous space, without villitis. PD-L1 expression in syncytiotrophoblast and Hofbaeur cells, together with limited production of NETs, may have prevented immune cell-driven placental damage, ensuring sufficient maternal-fetus nutrient exchanges.

(C) 2020 The Authors. Published by Elsevier B.V. This is an open access article under the CC BY-NC-ND license.
\end{abstract} (http://creativecommons.org/licenses/by-nc-nd/4.0/)
Funding. None.

* Corresponding author.

E-mail address: fabio.facchetti@unibs.it (F. Facchetti).

\# These authors equally contributed

\section{Introduction}

The COVID-19 pandemic has caused high levels of morbidity and mortality throughout the world, becoming a serious public health problem. The infection is caused by severe acute respiratory 


\section{Research in the context}

Evidence before this study

Several recent reports have addressed the question whether SARS-Cov-2 infection can be propagated from the mother to the fetus during pregnancy. Based on current data, evidence for vertical transmission of the SARS-CoV-2 infection is missing. Whereas detection of SARS-CoV-2 RNA in placental tissue has been recently documented, mechanisms limiting the spreading of virus infection to the newborn remain unknown. Distribution, composition and activation status of immune /inflammatory cells in placenta of COVID-19 pregnant women, remains undetermined.

Added value of this study

This study analyzed by immunohistochemistry the expression of SARS-CoV-2 Spike (S) protein in 101 placentas (including 15 from COVID-19 proved positive women) collected in Brescia, Italy during the SARS-Cov-2 pandemics. A single case showed robust expression of the $\mathrm{S}$ protein, as well as the $\mathrm{N}$ (nucleocapsid) protein in the syncytiotrophoblast. In addition, in situ hybridization for SARS-CoV-2 RNA demonstrated intense signal in the syncytiotrophoblast and in intervillous inflammatory cells, and ultrastructural analysis identified viral particles morphologically consistent with coronavirus localized in the cytoplasm of syncytiotrophoblast cells, in fetal capillaries endothelium, fibroblasts, as well as in fetal intravascular mononuclear cells. Histopathological analysis of the immune/ inflammatory cell infiltrate suggests that intervillous presence of activated monocyte-macrophages and neutrophils may represent an histological marker of SARS-CoV-2 infection of the placenta. This unique histopathological setting of the placenta coincided with early onset of COVID-19 disease in the newborn, consistent with vertical transmission of the infection.

Implications of all the available evidence

Together with previous reports, our study on 101 placentas from mothers collected in the COVID-19 pandemic stage documents that mother-to-fetus transmission of SARS-CoV-2 infection is a rare yet documented event. The findings provided in this study reveal the passage of SARS-CoV-2 across the maternal-fetal interface to infect fetal-derived cells of the placenta.

syndrome corona-virus 2 (SARS-CoV-2), a positive-sense RNA single-stranded virus, member of the Betacoronavirus genus that includes also SARS-CoV and MERS-CoV responsible of previous epidemic diseases.

The effects and consequences of COVID-19 on pregnant women and their infants remain poorly understood [1]. The adverse effects on women infected with SARS-CoV-2 during the first trimester are uncommon and may lead to early pregnancy loss [1,2]. Several recent reports describing individual cases or cohorts of COVID-19 women infected during the third trimester of pregnancy, have indicated that maternal complications are generally similar to those occurring in non-pregnant women [1-4], but an increased prevalence of preterm deliveries was noticed [2].

The increasing number of reports describing neonates born from women with COVID-19, with signs of early-onset infection $[1,3,5]$ is compatible with a scenario of trans-placental transmission of SARSCoV-2. However, vertical transmission of the virus remains controversial, with clinical and laboratory evidences considering it a rare event [2-4,6-10]. Notably, a cause-effect relationship between virus infection and unfavorable outcome of neonates has not been formally proven, as other factors causing post-partum complications cannot be excluded $[1,8]$. Therefore, uncertainty remains whether an early neonatal SARS-CoV-2 infection occurs in utero, during the delivery, or soon after birth [11].

Testing for SARS-CoV-2 presence by reverse transcriptase PCR has repeatedly failed to identify the presence of the viral genome in maternal and neonatal specimens including placenta, umbilical cord blood, amniotic fluid or amniotic swab, maternal blood, vaginal secretions and breastmilk [2,7,8,12,13].

The detection of SARS-CoV-2 RNA in placental or membrane samples, supporting the possibility of vertical transmission of the infection has been reported for the first time by Penfield et al.. [14] Authors detected viral RNA in 3 out of 11 samples from COVID-19 positive women, all presenting with moderate to severe disease at time of delivery. However, none of the infants tested positive for SARS-CoV-2 from postnatal day-1 through -5 , and none showed symptoms of COVID-19 infection.

Recently, two studies have provided further evidence for the presence of SARS-CoV-2 in the placenta, localizing it to syncytiotrophoblast cells at the maternal-fetal interface. In the study by Hosier et al. [15] a second-trimester pregnant woman with symptomatic COVID19 had severe preeclampsia and placental abruption. SARS-CoV-2 virus was detected in the placenta by molecular and immunohistochemical assays and by electron microscopy. Molecular analysis on tissue samples of the fetus obtained by autopsy resulted negative for the virus. In another study, two neonates born from symptomatic COVID-19 women resulted positive by nasopharyngeal swab testing immediately after birth and at day-7 post-partum, but none of them developed infection-related symptoms. The placenta from both cases was positive for SARS-CoV-2 by in situ hybridization and RT-PCR analysis [16].

Here, we report a case of a COVID-19 positive full-term pregnant woman with pneumonia and severe thrombocytopenia requiring childbirth induction. The neonate was positive for the presence of the virus and twenty-four hours after birth developed pneumonia and severe respiratory distress. SARS-CoV-2 viral products and/or particles were detected in villous syncytiotrophoblast, endothelial cells, fibroblasts and maternal macrophages contributing to the inflammatory infiltrate, as well as in Hofbauer cells. Remarkably, for the first time, particles morphologically consistent with coronavirus were localized in fetal circulating mononuclear cells.

In accordance with previous reports, the placental tissue contained an inflammatory infiltrate occupying the intervillous spaces. Comprehensive immunophenotyping analysis revealed that the infiltrate was composed of monocyte-macrophages displaying activation markers and both morphologically/phenotypically mature and immature neutrophils. Screening for SARS-CoV-2 proteins failed to identify positive cases among the remaining 100 placentas obtained from deliveries occurred during the pandemic period, which included 14 cases from COVID-19 positive mothers whose newborns tested negative. In summary, our observations provide proof for the occurrence at low frequency of intrauterine vertical transmission of SARSCoV-2 infection with clinical consequences for the newborn.

\section{Materials and methods}

\subsection{Study participants}

Brescia Spedali Civili Hospital is one among the Italian hospitals with the highest incidence of patients treated for COVID-19 infection. Starting from March 9, 2020, the Department of Obstetrics and Ginecology has been recognized as Regional Center for the handling of SARS-CoV-2 positive pregnant women. Between March 13 and May 4, SARS-CoV-2 RNA RT-PCR testing on nasopharyngeal swabs was selectively performed on women with positive clinical, laboratory or instrumental criteria. In particular, patients were tested for the presence of SARS-CoV-2 within 14 days of admission, upon presentation of at least one of the followings: fever $>37 \bullet 5^{\circ} \mathrm{C}$, cough, sore throat, 
rhinitis, dyspnea, ageusia or anosmia, diarrhea and/or vomit, myalgia, anamnesis of symptomatic relatives, close non protected contacts with COVID-19 confirmed cases, or when any of the following laboratory and instrumental tests at admission was present: lymphopenia, increase of transaminases unrelated to preeclampsia or gravidic hepatopathy, increased CRP, LDH, and pulmonary X-Ray or echography showing SARS-CoV-2 correlated interstitial pneumonia [17]. Starting May 5, all hospitalized patients were tested for SARS-CoV-2 RNA.

From February 7 to May 15, 2020101 placentas were collected, including 15 from women who tested positive for the SARS-CoV-2 RNA by RT-PCR, 34 tested negative for the presence of the virus, and 52 were not screened as they did not meet the above reported testing criteria (32), or delivered before COVID-19 pandemic declaration (20). We also included in the study 9 placentas examined in 2017 and 2018, showing respectively normal features (2 cases), cytomegalovirus infection (3) villitis (3) and villitis plus intervillositis (1).

The study was performed in accordance with Brescia Spedali Civili Hospital Institutional Ethical Board protocols and approved by the institutional review board (NP \#4151 COVID-BIO and NP \#4133 INTERCOVID). Written informed consent to pathology examination of the placenta and to its use for research-oriented testing was obtained from all participants, in accordance with the Declaration of Helsinki for Human Research.

\subsection{Clinical records}

The data of mothers and neonates of the COVID-19 positive and negative cases are reported in the supplementary Appendix. Details on the clinical history of the index case (Case \#1), including the evolution of pregnancy, the childbirth stages and support interventions, the clinical parameters of the newborn, and treatments applied to the mother and child are reported in Marzollo et al. [18].

Briefly, a 29 years-old woman was hospitalized at 37 weeks +5 of her first pregnancy, for fever $\left(38 \bullet 0 \mathrm{C}^{\circ}\right)$ and idiopathic thrombocytopenia, diagnosed at the beginning of the pregnancy. She tested positive for SARS-CoV-2 RNA and X-ray imaging showed parenchymal lung thickening (COVID-19 pneumonia 3/18 score) [17]. Due to fever and thrombocytopenia worsening, a vaginal delivery was induced, with supportive ventilation due to the onset of $95 \% 02$ saturation in room air. The male newborn weighted $2 \bullet 840 \mathrm{Kg}$ and presented an APGAR score of 9/10. Twenty-four hours post-partum he developed fever, breathing difficulty, vomiting, abdominal distension, hypotonia, and cutaneous mild erythema. Chest X-ray revealed interstitial pneumonia and he was transferred to intensive care unit, where mechanical ventilation was applied. After birth test for SARS-CoV-2 RNA on nasopharyngeal swab resulted inconclusive (amplification of less than three genes), while it was positive 36 and $72 \mathrm{~h}$ after birth and at the age of 17-days.

After delivery, the mother rapidly recovered, fever decreased and the puerperal course proceeded normally. The child was discharged on day-18 after birth in good general conditions. Two months after the delivery both mother and baby were healthy and asymptomatic.

\subsection{Pathological examination}

All placentas were fixed in $10 \%$ buffered formalin, weighed trimmed of cord and membranes and sampled according to the Amsterdam's guidelines [19]. The histological examination was performed by a pathologist experienced on fetal-placental pathology, who was not aware of the clinical diagnosis and COVID-19 status. The histological findings on placentas from the 15 COVID-19 positive women are reported in Table 1, whereas those on the 86 non COVID19 positive women are listed in Table $4 \mathrm{~s}$ of the Appendix.

All cases were stained by immunohistochemistry for the SARSCoV-2 S-protein using formalin fixed paraffin embedded sections. The 15 placentas from COVID-19+ ${ }^{+}$women were stained also for the
Table 1

Main morphological changes observed in the placentas from the 15 COVID-19 positive woman.

\begin{tabular}{|c|c|c|}
\hline CASE & $\begin{array}{l}\text { PLACENTAL WEIGHT (g)and } \\
\text { [percentile] }\left(^{*}\right)\end{array}$ & HISTOPATOLOGY $[19,40]$ \\
\hline 1 & $448[25-50]$ & $\begin{array}{l}\text { Histiocytic-neutrophilic intervil- } \\
\text { lositis } \\
\text { Avascular and fibrotic villi and } \\
\text { stroma-vascular karyorrhexis } \\
\text { Accelerated villous maturation } \\
\text { Chorangiosis }\end{array}$ \\
\hline 2 & $472[25-50]$ & $\begin{array}{l}\text { Borderline massive perivillous } \\
\text { fibrin deposition }\end{array}$ \\
\hline 3 & $440[25-50]$ & $\begin{array}{l}\text { Maternal vessels thrombosis of } \\
\text { capsularis decidua } \\
\text { Focal acute chorioamnionitis }\end{array}$ \\
\hline 4 & $240[5-10]$ & $\begin{array}{l}\text { Infarct } \\
\text { Intervillous hematoma }\end{array}$ \\
\hline 5 & $435[25-50]$ & Normal \\
\hline 6 & $429[75-90]$ & Maternal decidual vasculopathy \\
\hline 7 & $542[55-75]$ & $\begin{array}{l}\text { Chorionic plate infarct } \\
\text { Borderline massive perivillous } \\
\text { fibrin deposition }\end{array}$ \\
\hline 8 & $400[10-25]$ & $\begin{array}{l}\text { Small infarcts } \\
\text { Acute deciduitis } \\
\text { Focal distal villous immaturity }\end{array}$ \\
\hline 9 & $444[25-50]$ & $\begin{array}{l}\text { Transmural massive perivillous } \\
\text { fibrin deposition } \\
\text { Infarct and acute deciduitis } \\
\text { Accelerated villous maturation }\end{array}$ \\
\hline 10 & $515[50-75]$ & $\begin{array}{l}\text { Chorionic vasculitis } \\
\text { Intervillous hematomas } \\
\text { Chorangiosis } \\
\text { Infarct } \\
\text { Peripheral funisitis }\end{array}$ \\
\hline 11 & $470[25-50]$ & $\begin{array}{l}\text { Transmural massive perivillous } \\
\text { fibrin deposition } \\
\text { Intervillous hematomas } \\
\text { Chorangiosis }\end{array}$ \\
\hline 12 & $790[>90]$ & $\begin{array}{l}\text { Accelerated villous maturation } \\
\text { Subchorial hematoma }\end{array}$ \\
\hline 13 & 582 [75-90] & $\begin{array}{l}\text { Accelerated villous maturation } \\
\text { Intervillous hematomas } \\
\text { Chorangiosis }\end{array}$ \\
\hline 14 & $440[25]$ & Marginal infarct \\
\hline 15 & $477[25-50]$ & $\begin{array}{l}\text { Chronic villitis and histiocytic- } \\
\text { lymphocytic intervillositis } \\
\text { Maternal infarct } \\
\text { Accelerated villous maturation }\end{array}$ \\
\hline
\end{tabular}

* Percentiles and standard deviations for placental weights by gestational age.

SARS-CoV-2 N-protein. For the single placenta tested positive for SARS-CoV-2 proteins, the inflammatory infiltrate as well as other cell types of the placenta were studied using a comprehensive set of antibodies tested in single or double immunostains. Immunophenotyping was extended to the inflammatory infiltrate of placentas showing villitis associated or not with an intervilllous inflammatory component (Case 15, 29, 32, 53, 61, 63, 99 and 101). The list of antibodies used and their specificities are reported in the Appendix. Placenta from Case \#1 tested positive for SARS-CoV-2, after detecting the presence of S-protein-specific transcripts by RNA-in situ hybridization. Sections were subsequently immunostained to detect the expression of the CD14 antigen. SARS-CoV-2 RNA RT-PCR analysis, immunofluorescence microscopy to identify Neutrophil Extracellular Traps (NETs), negative stain electron microscopy and transmission electron microscopy were performed as reported in the Appendix.

\subsection{Statistical analysis}

Hofbauer cell number in placenta from Case \#1 was compared to that of a reference group of controls. For analysis of changes, twotailed paired $t$-test was applied. 


\section{Results}

\subsection{Histological, immunohistochemical and in situ SARS-CoV-2 RNA analyses of placenta}

The monoamniotic-monocorial placenta of Case \#1 weighted 448 gr (25-50 percentiles for age). No significant abnormalities were detected on cord insertion, cord vessels, fetal surface and membranes, while the dark brown colored chorionic parenchyma contained multiple small pale areas (Appendix). On microscopical examination, the chorionic plate on both fetal and maternal sites showed multiple foci (corresponding to around $40 \%$ of the total surface) with intervillous inflammatory infiltrate, composed of numerous monocytoid cells and macrophages and mature neutrophils. In areas showing inflammation, villi displayed variable changes compatible with fetal vascular malperfusion with avascular villi and stroma-vascular karyorrhexis. Fibrin perivillous deposition was prominent in many areas. In the remaining parenchyma normal villi and focal chorangiosis were observed. No intra-villous inflammatory infiltrate (villitis) was evident (Appendix)

Immunostains for SARS-CoV-2 proteins showed positivity in the cytoplasm of the syncytiotrophoblast for both S-protein and N-protein (Fig. 1A and B). N-protein was also expressed by rare intervillous macrophages and Hofbauer cells. Notably, while anti-nucleocapsid staining was homogenously strong throughout the placental parenchyma, the immunoreactivity to S-protein was variable and particularly strong in areas with abundant intervillous inflammation (Fig. 1C). SARS-CoV-2 proteins were not detected in villous capillaries.

Immunophenotyping showed a heterogeneous composition of inflammatory cells, which mostly consisted of monocyte-macrophages and neutrophils (Fig. 1D). The former strongly expressed CD11c, CD14, CD68, and CD163. Most of them were also positive for CD16, and about 10\% co-expressed myeloperoxidase (Appendix), while CD66b was negative. Stain for PD-L1 showed weak to moderate positivity in a proportion of these cells (Fig. 1E). The neutrophils subsets included both mature cells expressing CD10, CD11c, CD15, CD16, myeloperoxidase, CD66b and Arginase markers, and immature cells with monocytoid morphology lacking CD10 expression [20] (Appendix).

Notably, the comparison between Case \#1 and the remaining placentas, including the fourteen from COVID-19-positive women, did not show significant morphological differences, except for the prominent intervillous inflammation and its mixed monocyte-macrophage and neutrophil composition. Furthermore, all other cases showing variable degree of inflammation in the intervillous area were associated with villitis and the presence of $\mathrm{CD} 68^{+} \mathrm{CD} 14^{+} \mathrm{CD} 163^{+}$monocytemacrophages and scattered $\mathrm{CD}^{+} \mathrm{T}$-lymphocytes, while very rare $\mathrm{CD} 6 \mathrm{~b}^{+}$neutrophils were detected (Appendix).

In light of the high number of neutrophils in Case \#1, we tested by immunofluorescence microscopy the presence of Neutrophil Extracellular Traps (NETs)(Appendix). We observed a high density of nuclear debris marked by DAPI and citrullinated Histone H3 (H3cit), but only rare myeloperoxidase-positive cells in which a H3cit/DAPI thread was detected, thus indicating a limited representation of NETosis (Appendix).

The absence of intravillous inflammation was confirmed by immunostains for markers identifying different T-cell subsets, B cells, NK cells and plasmacytoid dendritic cells (Appendix). Notably Hofbauer macrophages were significantly increased in number (Fig. 1D and Appendix) and, in areas of inflammation, expressed PD-L1, which is otherwise absent on these cells (Fig. 1E). The Hofbauer cell hyperplasia was confirmed by comparing the number of $\mathrm{CD} 163^{+}$cells in terminal villi in the SARS-CoV- $2^{+}$placenta (average $5 \bullet 7 \pm 4 \bullet 5$ SD cells per villous) to those of sixteen control samples, including cases with inflammation unrelated to SARS-CoV-2 $(n=10$ and $n=6$ from
COVID-19 positive and negative patients, respectively; $3 \bullet 9 \pm 2 \bullet 5 /$ villous SD)(t-test for independent means: $p<0 \bullet 001$ ). The difference was stronger when we evaluated only villi displaying inflammation in the SARS-CoV-2 positive placenta (6.7 cells/villous).

In areas of inflammation we found prominent platelet deposition revealed by antibodies recognizing the megakaryocyte and platelet associated antigens CD61, LAT/Linker for Activation of T-cells and CXCR4 (Appendix). In placentas from six control cases, platelet aggregates were rare and mostly located on the contour of stem villi. Notably, staining with the three antibodies also identified villous intracapillary megakaryocytes (Appendix), whose number in the SARSCoV-2 positive case was three times higher than in seven controls $\left(4 \bullet 7\right.$ versus $1 \bullet 4$ per $\mathrm{mm}^{2}$ of parenchyma). In rare capillaries, loose aggregates of platelets but no fibrin thrombi were found.

In keeping with evidence of syncytiotrophoblast damage in villi associated with the intervillous inflammatory infiltrate, we found thinning, or even discontinuity of this cell layer by cytokeratin stain (Appendix). Interestingly, in the same areas, the syncytiotrophoblast was positive for PD-L1 staining, with a less defined expression pattern compared to non-inflamed areas (Fig. 1E).

\subsection{RNA in situ hybridization}

SARS-CoV-2 RNA in situ hybridization (ISH) using the S antisense probe showed intense signal positivity in the syncytiotrophoblast, with a distribution similar to that detected for the S-protein by immunohistochemistry (Fig. 1F). SARS-CoV-2 S-specific transcripts tested positive also in scattered cells in the intervillous areas, most of which were represented by CD14-expressing monocytes-macrophages (Fig. 1F).

\subsection{Electron Microscopy (EM)}

Electron microcopy of formalin-fixed placental tissue revealed fine morphologic preservation. On negative stain EM (nsEM), we observed particles morphologically compatible with coronavirus. They consisted of round particles with an external "corona" made of peplomers, and their size ranged between 85 and $90 \mathrm{~nm}$ to $110-120 \mathrm{~nm}$ (including peplomers). Isolated coronavirus but also couples or small clusters of particles were identified (Fig. 2A and Appendix).

On ultrathin sections coronavirus resembling particles were easily identified by their mostly spherical shape, with intermediate electron density cores, often surrounded by an envelope covered with faint surface projections (peplomeric spikes). The diameter of these particles was similar to the one measured by nsEM, and consistent with size and appearance of both SARS-CoV [21] and SARSCoV-2 (Fig. 2B) [22]. Viral particles localized predominantly in the cytoplasm of syncytiotrophoblast cells as single particle or small groups, either dispersed or within small vesicles. Moreover, electron-dense granular material corresponding to nucleocapsid inclusions were found (Fig. 2C1-C2) [21]. In addition to the syncytiotrophoblast localization, virus particles were identified in the endothelium of fetal capillaries close to the villus surface (Fig. 2 C3-C4 and Fig. 2D-D1) and in fibroblasts. Importantly, particles morphologically consistent with coronavirus were detected in the cytoplasm of intra-capillary cells, likely corresponding to monocytes (Fig. 3A-B), indicating a possible role of these cells in the spread of the infection to the fetus.

\section{Discussion}

In this study we provide evidence in support of transmission of the SARS-CoV-2 infection from a COVID-19 full term pregnant woman to her newborn, who resulted positive for SARS-CoV-2 and developed pneumonia soon after birth. Viral proteins and RNA were 

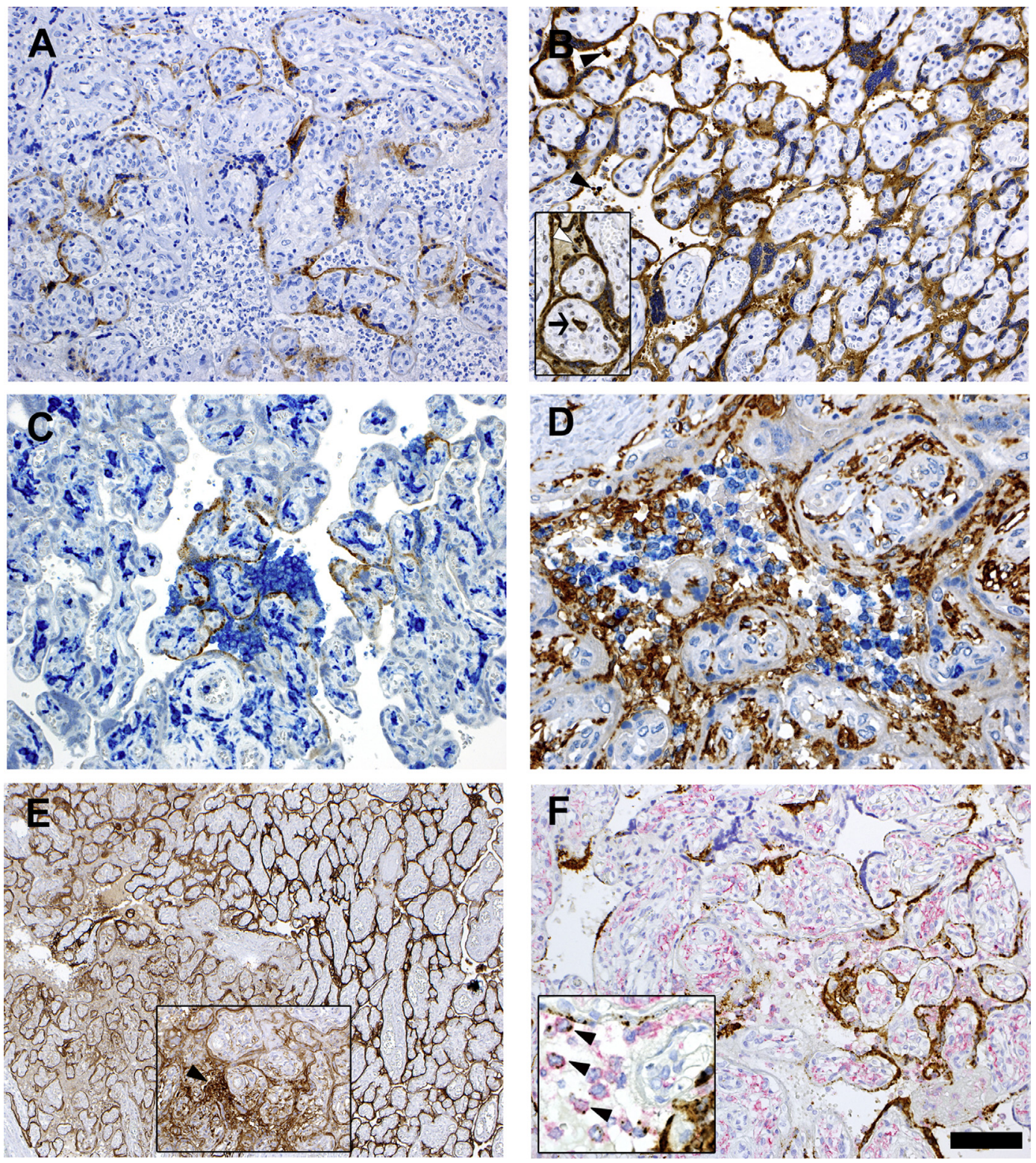

Fig. 1. Immunohistochemical and RNA-in situ stains.

(A, B) Immunostainings for SARS-CoV-2 spike (A) and nucleocapsid (B) proteins. Syncytiotrophoblast is positive for both proteins. Note the more extensive and stronger positivity for nucleocapsid expression, which also localizes to intervillous inflammatory cells (inset, arrowheads) and an Hofbauer cell (inset, arrow).

(C) Double stain for spike protein (brown) and CD14 (blue) indicates higher density of inflammatory infiltrate at sites where the viral protein is more intensely expressed.

(D) Double staining for CD14 (brown) and CD66b (blue) expression, respectively identifying monocyte-macrophages and neutrophils in the inflammatory infiltrate occupying the intervillous spaces. Note the CD14-positive Hofbauer cells in the villi.

(E) PD-L1 staining shows strong expression in the syncytiotrophoblast in areas displaying inflammation (left side). The inset shows an area infiltrated with inflammatory cells, which express PD-L1 (arrow), similarly to intravillous Hofbauer cells.

(F) SARS-CoV-2 RNA in situ hybridization using the S antisense probe (brown stain) combined with anti-CD14 immunostain (red stain). Strong expression of viral RNA is

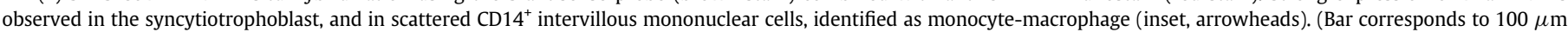
for figs. A-B-B inset-C-F, $50 \mu \mathrm{m}$ for fig. D and F inset, $200 \mu \mathrm{m}$ for fig. E and $150 \mu \mathrm{m}$ for E inset).

detected in multiple fetal villous cellular subsets and in maternal inflammatory cells. Moreover, particles consistent with coronavirus were identified by electron microscopy in villous syncytiotrophoblast, endothelial cells, fibroblasts, and, most notably, fetal intravascular monocytes. Infection by SARS of infiltrating monocytes and macrophages has been documented in human tissues [23,24]. The hitherto unreported presence of SARS-CoV-2 in circulating cells is in keeping with the detection of SARS-CoV-2 RNA in blood samples from COVID-19 patients [25]. These results raise the hypothesis that fetal blood cells infected with SARS-CoV-2 represent a vehicle of the infection.
Fetal placental site infection by SARS-CoV-2 has been recently reported in other three cases, but no SARS-CoV-2 RNA was detected in fetal tissues [15] and neither of the two newborns developed SARS-CoV-2 related disease [16]. As previously shown, we detected viral S-protein and RNA in villous syncytiotrophoblast. We found stronger expression of S-protein in areas with dense monocyte-macrophage inflammation, compatible with a local activation of these immune cells in response to the binding to anti-Spike maternal IGG-virus immune complexes, as shown in lung tissues [26]. A broader and stronger positivity for SARS-CoV$2 \mathrm{~N}$-protein was detected in the same cells and in maternal and 

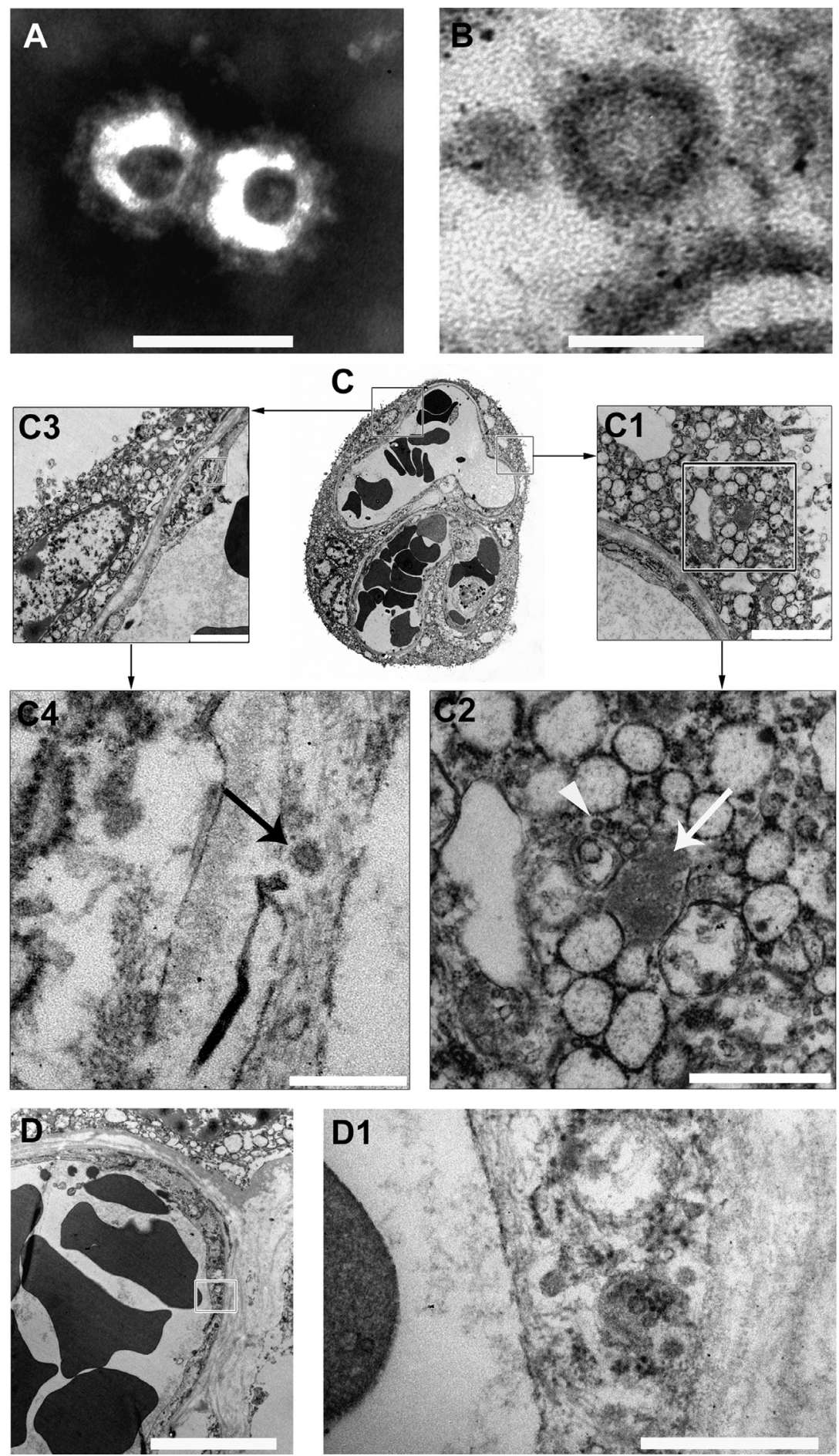

Fig. 2. Electron microscopy study of placenta.

(A, B) Negative stain electron micrograph of particles obtained from a homogenate of formalin-fixed placenta (A, bar $100 \mathrm{~nm})$ and ultrathin sections of free particle in the cytoplasm of a syncytiotrophoblast cell (B, bar $100 \mathrm{~nm}$ ). In both cases morphological features consistent with coronavirus are observed.

(C) Ultrathin sections of a villous. The right and the left squares include a portion of the syncytiotrophoblast and of a capillary vessel; the respective details are illustrated in fig.

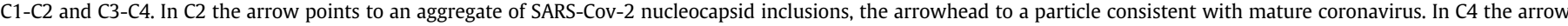
points to a particle with the typical morphological features of coronavirus within the endothelium. (C1, bar $2 \mu \mathrm{m} ; \mathrm{C} 2$, bar $1 \mu \mathrm{m}$; C3, bar $2 \mu \mathrm{m}$; $\mathrm{C} 4$, bar $250 \mathrm{~nm}$ ).

(D, D1) The endothelium of a fetal capillary (D, bar $5 \mu \mathrm{m}$ ) close to the villus surface shows particles morphologically consistent with SARV-CoV-2 in the cytoplasm (D1, high magnification of the area shown in C, bar $500 \mathrm{~nm}$ ).

fetal villous macrophages. This observation is in agreement with evidence that the $\mathrm{N}$-protein can be produced twice as much as the $S$ protein in Coronavirus-infected cells [27-30], even in the absence of complete virus particle assembly [21,22], as witnessed in the syncytiotrophoblast cells.
Whereas our findings cannot rule out ongoing clearance of viral particles or infected cells by macrophages [27], the strong positivity for SARS-CoV-2 N-protein in these antigen-presenting cells is compatible with potent immunogenicity conferred by this protein to different SARS viruses [28]. Indeed, a restricted IGG-specific anti-N 

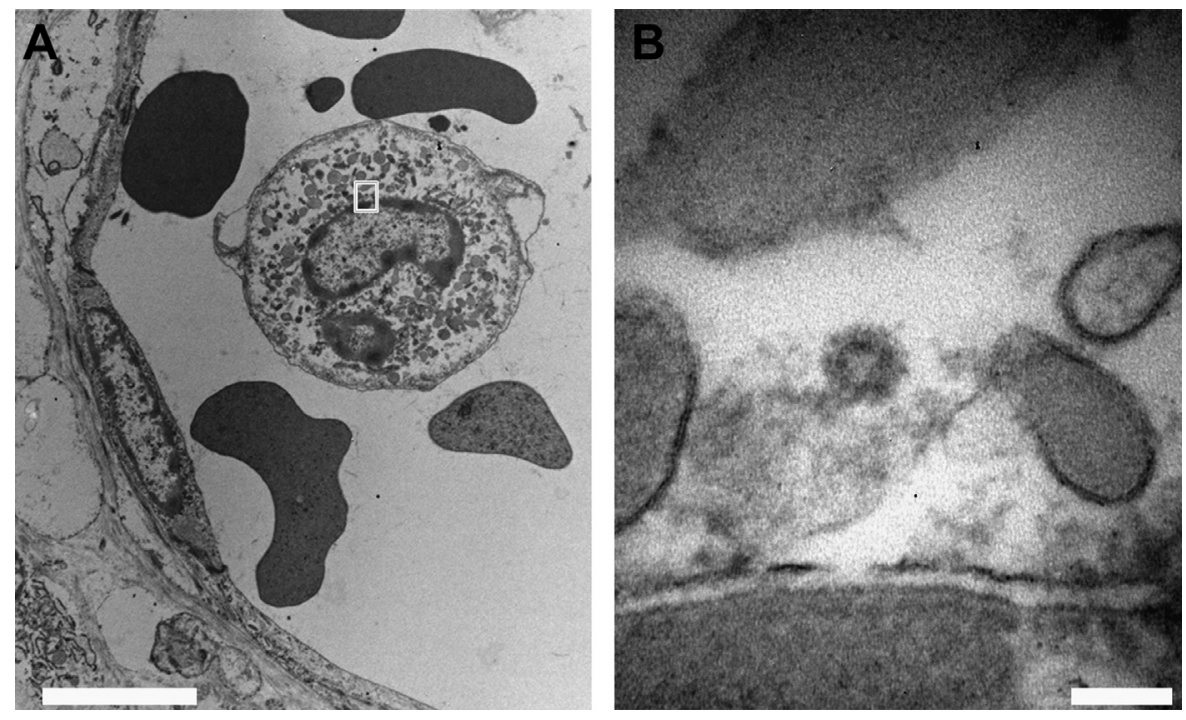

Fig. 3. Electron microscopy of SARS-CoV-2 viral particle within an intra-capillary monocyte.

(A, B) In the cytoplasm of an intra-capillary monocyte (A, bar $5 \mu \mathrm{m}$ ) a particle morphologically consistent with coronavirus is present (B, high magnification of the area shown in A, bar $100 \mathrm{~nm})$.

antibody response [31] was recently shown to exert an important role in host immunity in COVID-19 patients [27,32].

Despite chorionic villi associated with intervillous inflammation and significant cell damage contained virus particles in different cell types, there was no evidence of active recruitment of intravillous inflammatory cells. The only sign of local inflammatory activation was linked to Hofbauer cell hyperplasia. Similar findings were reported in studies of placenta of fetuses with congenital Zika virus infection [33], where infected Hofbauer cells were proposed to eliminate viruses by secreting type-I interferon and by upregulating antiviral genes [34]. Despite the lack of definite explanations for the missed recruitment of inflammatory cell to the villi, it may depend on de novo expression of the check-point inhibitor PD-L1 by Hofbauer cells and the syncytiotrophoblast.

The two previous studies on SARS-CoV-2 infected placentas defined the intervillous inflammatory infiltrate as "chronic histiocytic intervillositis" [35] on the basis of the single CD68 marker. In the present study, using a comprehensive set of markers, we describe instead a composite pool of inflammatory cell types, consisting of monocyte-macrophages and mature and immature neutrophils. Notably, a proportion of the monocyte-macrophages cells expressed PD-L1 in combination with myeloperoxidase, indicating an activated state [36]. The presence of a substantial pool of monocytic-macrophages and neutrophils is uncommon in normal and pathological placental conditions, with the exception of so called "immunological" preeclampsia [37], where intervillous neutrophils are associated with high triggering of Neutrophil Extracellular Traps (NETs) and severe tissue damage [38]. Future studies will establish whether the presence of a mixed monocytic-macrophage and neutrophil infiltrate represents an histological marker of SARS-CoV-2 placenta infection. Aberrant NETs formation has been recently observed in lungs autopsy specimens from COVID-19 patients with neutrophil-rich inflammation, suggesting a role for NETs in the development of complications of SARS-CoV-2 infection [39]. Immunofluorescence microscopy analyses of the placenta of COVID-19 Case \#1 revealed a high density of nuclear debris marked by DAPI and citrullinated Histone H3 (H3cit). However, only rare myeloperoxidase-positive cells with a H3cit/DAPI thread were detected, thus pointing to a minor contribution of NETosis to the inflammatory response ongoing in SARS-CoV-2-infected placental tissue.

In conclusion, the detection of SARS-CoV-2 in the placenta of COVID-19 Case \#1, distributed in multiple fetal cell types, including circulating cells, combined with the failure to reveal SARS-CoV-2 S-protein expression in remaining 100 placentas from deliveries dating to the pandemic period, including 14 from women tested positive for COVID-19, establishes the infrequent occurrence of mother-tofetus SARS-Cov-2 transmission with adverse effects on the newborn. Whether the rare permissiveness of trans-placental SARS-CoV-2 transmission seen in COVID-19 Case \#1 depended on host genetic factor(s), the infection with a unique SARS-CoV-2 genetic variant, and/or the production of a high load of maternal antibody-virus immune complexes contributing to antibody-dependent enhancement of the infection awaits future investigations.

\section{Contributors}

$\mathrm{FF}, \mathrm{MB}$ were responsible for the design of the study and the writing of the manuscript.

$\mathrm{FF}, \mathrm{MB}, \mathrm{CT}, \mathrm{AL}, \mathrm{SC}$ were responsible for the analysis and interpretation of the data.

AL, SC contributed to the writing of the manuscript.

$\mathrm{MB}, \mathrm{CT}, \mathrm{ED}, \mathrm{VC}, \mathrm{MBB}, \mathrm{PC}, \mathrm{AL}$ were responsible for the laboratory analysis.

ES, MP, RC were responsible for the collection of clinical data of the patients.

All authors reviewed and approved the final version of the manuscript.

\section{Declaration of interest}

all authors declare no competing interests.

\section{Acknowledgments}

The Authors are grateful to Giovanni Bozzoni, T.h.S., and Moris Cadei T.h.S. for the preparation of electron microscopical specimens.

\section{Supplementary materials}

Supplementary material associated with this article can be found, in the online version, at doi:10.1016/j.ebiom.2020.102951. 


\section{References}

[1] Schwartz DA, Dhaliwal A. Infections in pregnancy with COVID-19 and other respiratory RNA virus diseases are rarely, if ever, transmitted to the fetus: experiences with coronaviruses, HPIV, HMPV RSV, and Influenza. Arch Pathol Lab Med 2020.

[2] Yan J, Guo J, Fan C, Juan J, Yu X, Li J, et al. Coronavirus disease 2019 (COVID-19) in pregnant women: a report based on 116 cases. Am J Obstet Gynecol 2020;223 111.e1-e14.

[3] Zaigham M, Andersson O. Maternal and perinatal outcomes with COVID-19: a systematic review of 108 pregnancies. Acta Obstet Gynecol Scand 2020;99:823-9.

[4] Yang Z, Wang M, Zhu Z, Liu Y. Coronavirus disease 2019 (COVID-19) and pregnancy: a systematic review. J Matern Fetal Neonatal Med 2020:1-4.

[5] Alzamora MC, Paredes T, Caceres D, Webb CM, Valdez LM, La Rosa M. Severe COVID-19 during Pregnancy and Possible Vertical Transmission. Am J Perinatol 2020;37:861-5

[6] Zhu H, Wang L, Fang C, Peng S, Zhang L, Chang G, et al. Clinical analysis of 10 neonates born to mothers with 2019-nCoV pneumonia. Transl Pediatr 2020;9:51-60.

[7] Liu W, Wang J, Li W, Zhou Z, Liu S, Rong Z. Clinical characteristics of 19 neonates born to mothers with COVID-19. Front Med 2020;14:193-8.

[8] Schwartz DA. An analysis of 38 Pregnant Women with COVID-19, their newborn infants, and Maternal-Fetal transmission of SARS-CoV-2: maternal Coronavirus infections and pregnancy outcomes. Arch Pathol Lab Med 2020.

[9] Baergen RN, Heller DS. Placental Pathology in Covid-19 positive mothers: preliminary findings. Pediatr Dev Pathol 2020;23:177-80.

[10] Shanes ED, Mithal LB, Otero S, Azad HA, Miller ES, Goldstein JA. Placental Pathology in COVID-19. Am J Clin Pathol 2020;154:23-32.

[11] Zeng L, Xia S, Yuan W, Yan K, Xiao F, Shao J, et al. Neonatal Early-Onset Infection With SARS-CoV-2 in 33 Neonates born to mothers with COVID-19 in Wuhan, China. JAMA Pediatr 2020;174:722-5.

[12] Yang P, Wang X, Liu P, Wei C, He B, Zheng J, et al. Clinical characteristics and risk assessment of newborns born to mothers with COVID-19. J Clin Virol 2020;127.

[13] Chen H, Guo J, Wang C, Luo F, Yu X, Zhang W, et al. Clinical characteristics and intrauterine vertical transmission potential of COVID-19 infection in nine pregnant women: a retrospective review of medical records. Lancet 2020;395:809-15.

[14] Penfield CA, Brubaker SG, Limaye MA, Lighter J, Ratner AJ, Thomas KM, et al. Detection of SARS-COV-2 in placental and fetal membrane samples. Am J Obstet Gynecol MFM 2020.

[15] Hosier H, Farhadian SF, Morotti RA, Deshmukh U, Lu-Culligan A, Campbell KH, et al. SARS-CoV-2 infection of the placenta. J Clin Invest 2020.

[16] Patane L, Morotti D, Giunta MR, Sigismondi C, Piccoli MG, Frigerio L, et al. Vertical transmission of COVID-19: sARS-CoV-2 RNA on the fetal side of the placenta in pregnancies with COVID-19 positive mothers and neonates at birth. Am J Obstet Gynecol MFM 2020.

[17] Borghesi A, Maroldi R. COVID-19 outbreak in Italy: experimental chest X-ray scoring system for quantifying and monitoring disease progression. Radiol Med 2020;125:509-13.

[18] Marzollo R, Aversa S, Prefumo F, Saccani B, Rodriguez-Perez C, Sartori E, Motta M. COVID-19 pandemic and pregnancy: vertical transmission is not excluded. Pediatr Infect Dis J 2020 (in press).

[19] Khong TY, Mooney EE, Ariel I, Balmus NC, Boyd TK, Brundler MA, et al. Sampling and definitions of Placental Lesions: amsterdam Placental workshop group consensus statement. Arch Pathol Lab Med 2016;140:698-713.

[20] Marini O, Costa S, Bevilacqua D, Calzetti F, Tamassia N, Spina C, et al. Mature CD10 $(+)$ and immature CD10 (-) neutrophils present in G-CSF-treated donors display opposite effects on T cells. Blood 2017;129:1343-56.
[21] Goldsmith CS, Tatti KM, Ksiazek TG, Rollin PE, Comer JA, Lee WW, et al. Ultrastructural characterization of SARS coronavirus. Emerg Infect Dis 2004:10:320-6.

[22] Farkash EA, Wilson AM, Jentzen JM. Ultrastructural evidence for direct renal infection with SARS-CoV-2. J Am Soc Nephrol 2020.

[23] Menter T, Haslbauer JD, Nienhold R, Savic S, Hopfer H, Deigendesch N, et al. Postmortem examination of COVID19 patients reveals diffuse alveolar damage with severe capillary congestion and variegated findings of lungs and other organs suggesting vascular dysfunction. Histopathology 2020.

[24] Gu J, Gong E, Zhang B, Zheng J, Gao Z, Zhong Y, et al. Multiple organ infection and the pathogenesis of SARS. J Exp Med 2005;202:415-24

[25] Huang C, Wang Y, Li X, Ren L, Zhao J, Hu Y, et al. Clinical features of patients infected with 2019 novel coronavirus in Wuhan, China. Lancet 2020;395:497506.

[26] Liu L, Wei Q, Lin Q, Fang J, Wang H, Kwok H, et al. Anti-spike IgG causes severe acute lung injury by skewing macrophage responses during acute SARS-CoV infection. JCI Insight 2019;4.

[27] Merad M, Martin JC. Pathological inflammation in patients with COVID-19: a key role for monocytes and macrophages. Nat Rev Immunol 2020;20:355-62.

[28] McBride R, van Zyl M, Fielding BC. The coronavirus nucleocapsid is a multifunctional protein. Viruses 2014;6:2991-3018.

[29] Li YH, Li J, Liu XE, Wang L, Li T, Zhou YH, et al. Detection of the nucleocapsid protein of severe acute respiratory syndrome coronavirus in serum: comparison with results of other viral markers. J Virol Methods 2005;130:45-50.

[30] Wang J, Ji J, Ye J, Zhao X, Wen J, Li W, et al. The structure analysis and antigenicity study of the N protein of SARS-CoV. Genomics Proteomics Bioinform 2003: $1: 145-54$.

[31] Leung DT, Tam FC, Ma CH, Chan PK, Cheung JL, Niu H, et al. Antibody response of patients with severe acute respiratory syndrome (SARS) targets the viral nucleocapsid. J Infect Dis 2004;190:379-86.

[32] Zeng W, Liu G, Ma H, Zhao D, Yang Y, Liu M, et al. Biochemical characterization of SARS-CoV-2 nucleocapsid protein. Biochem Biophys Res Commun 2020;527: 618-23.

[33] Schwartz DA. Viral infection, proliferation, and hyperplasia of Hofbauer cells and absence of inflammation characterize the placental pathology of fetuses with congenital Zika virus infection. Arch Gynecol Obstet 2017;295:1361-8.

[34] Zulu MZ, Martinez FO, Gordon S, Gray CM. The Elusive Role of Placental Macrophages: the Hofbauer Cell. J Innate Immun 2019;11:447-56.

[35] Bos M, Nikkels PGJ, Cohen D, Schoones JW, Bloemenkamp KWM, Bruijn JA, et al, Towards standardized criteria for diagnosing chronic intervillositis of unknown etiology: a systematic review. Placenta 2018;61:80-8.

[36] Rodrigues MR, Rodriguez D, Russo M, Campa A. Macrophage activation includes high intracellular myeloperoxidase activity. Biochem Biophys Res Commun 2002;292:869-73.

[37] Leavey K, Grynspan D, Cox BJ. Both "canonical" and "immunological" preeclampsia subtypes demonstrate changes in placental immune cell composition. Placenta 2019;83:53-6.

[38] Marder W, Knight JS, Kaplan MJ, Somers EC, Zhang X, O'Dell AA, et al. Placental histology and neutrophil extracellular traps in lupus and pre-eclampsia pregnancies. Lupus Sci Med 2016;3.

[39] Barnes BJ, Adrover JM, Baxter-Stoltzfus A, Borczuk A, Cools-Lartigue J, Crawford JM, et al. Targeting potential drivers of COVID-19: neutrophil extracellular traps. J Exp Med 2020;217.

[40] Ernst LM, Sheata BM, McKay E, Chisholm K, Katzman P, Linn R, et al. In: Chance D Geisinger A, Green Terry A, Conno S, Cannon T, Cronin J, editors. Philadelphia, PA: Elsevier; 2015. 